\title{
INTELIGENCIA EMOCIONAL EN MAESTROS DE EDUCACIÓN PRIMARIA: UNA PROPUESTA PRÁCTICA PARA SU DESARROLLO
}

\author{
EMOTIONAL INTELLIGENCE IN PRIMARY EDUCATION \\ TEACHERS: A PRACTICAL PROPOSAL FOR ITS \\ DEVELOPEMENT
}

\begin{abstract}
ב"
Raúl Campayo-Julve $e^{a}$ Martín Sánchez-Gómez ${ }^{a^{*}}$
\end{abstract}

Fechas de recepción y aceptación: 17 de junio de 2020 y 2 de septiembre de 2020

DOI: https://doi.org/10.46583/edetania_2020.58.689

Resumen: A finales del siglo xx varias aportaciones desde la psicología significaron un cambio de paradigma en la educación. Una de las más relevantes fue la inteligencia emocional, término acuñado por los psicólogos Salovey y Mayer en 1990. Durante estas tres décadas, numerosas investigaciones evidencian el impacto de la inteligencia emocional en distintos ámbitos como el educativo, lo que se ha traducido en el desarrollo y puesta en práctica de diversos programas de desarrollo emocional que han demostrado su efectividad. El presente trabajo realiza una investigación bibliográfica cualitativa a partir de la que se propone un programa pedagógico dirigido a docentes de tercer ciclo de primaria para que puedan formar a sus alumnos en esta habilidad. Se concluye que la inteligencia emocional es un factor de gran relevancia en educación para el desarrollo personal y socioemocional de los alumnos, y se proponen distintas actividades para que los docentes pongan en práctica programas centrados en el desarrollo de la IE.

Palabras clave: inteligencia emocional, educación, profesorado, modelo de habilidad, educación primaria.

${ }^{a}$ Departamento de Psicología Evolutiva, Educativa, Social y Metodología. Facultad de Ciencias de la Salud. Universitat Jaume I.

${ }^{*}$ Correspondencia: Universitat Jaume I. Facultad de Ciencias de la Salud. Departamento de Psicología Evolutiva, Educativa, Social y Metodología. Avenida Vicent Sos Baynat, s/n. 12071 Castellón de la Plana. España.

E-mail: sanchgom@uji.es 
Abstract: At the end of the $20^{\text {th }}$ century several contributions from psychology helped to create a paradigm shift in education. Emotional intelligence, term coin by the psychologists Salovey and Mayer in 1990, was one of the most relevant contributions. During these three decades, an enormous number of investigations have been carried out evidencing the impact of emotional intelligence in different areas such as education, which has meant the creation and implementation of various emotional development programs that have demonstrated their effectiveness. The present work, based on a qualitative bibliographic research, proposes a pedagogical program to primary school teachers to give their students the possibility of developing this ability. It is concluded that emotional intelligence is a factor of great relevance in education for the personal and socioemotional development of students, and are different activities are proposed to implement programs focused on the development of EI.

Keywords: emotional intelligence, education, teachers, ability model, primary education.

\section{INTRODUCCIÓN}

\subsection{Justificación}

El momento histórico en el que nos encontramos plantea un complejo escenario en el que llevar a cabo la tarea de educar (Camusso, 2019). El sistema educativo juega un papel fundamental en la preparación de los estudiantes, por ello debe entender las necesidades y requerimientos de la sociedad para poder ofrecer al estudiante los recursos necesarios para desenvolverse en ella de la mejor forma posible. En este proceso la figura del docente juega un papel fundamental, especialmente en educación primaria, una etapa marcada por los importantes cambios biopsicosociales. Según Elias, Arnold y SteigerHussey (2003), el propósito del maestro en las primeras etapas educativas es, además de enseñar su materia, ayudar a los alumnos a desarrollar habilidades socioemocionales. El docente es el agente más relevante en el aula para determinar el éxito de los estudiantes, por ello, es importante que sea capaz de entender y conocer a los alumnos y, a su vez, debe ser capaz de enseñarles a desarrollar destrezas para adaptarse al contexto (Vallés, 2001).

Las investigaciones realizadas hasta la fecha han señalado que los entornos educativos que promueven el desarrollo socioemocional preparan mejor a los estudiantes para la educación postsecundaria y el mundo laboral, generando así un retorno positivo en las inversiones (Belfield et al., 2015). Weissberg, Durlak, Domitrovich y Gullotta (2015) señalan que tanto estudiantes como 
adultos necesitan aprender y practicar habilidades socioemocionales, tanto en instrucción formal como en entrenamiento informal. Por ende, parece necesario formar a los alumnos en habilidades emocionales mediante programas con metodologías que partan de un modelo teórico claro, específico y válido. De tal modo, y sabiendo que las competencias emocionales son una necesidad que avanza en la actualidad, se propone un programa para desarrollar la inteligencia emocional en el alumnado de último ciclo de educación primaria.

\subsection{Una nueva forma de entender la inteligencia}

La aparición, en primer lugar, de la teoría de las inteligencias múltiples de Howard Gardner (1983) y, posteriormente, del concepto de inteligencia emocional (IE) (Salovey y Mayer, 1990) ayudó a generar un cambio de paradigma a nivel educativo en el que la inteligencia se contempla de un nuevo modo. Tradicionalmente, la educación se centraba en aquellas habilidades o inteligencias propiamente académicas, medidas por los test de inteligencia (CI), los cuales en cierto modo predecían el futuro éxito académico y profesional. Sin embargo, estas teorías abren un nuevo horizonte educativo en el que se empieza a reflexionar sobre las habilidades que deben ser desarrolladas para conseguir que los alumnos desplieguen y potencien al máximo su talento, orientándoles así hacia el éxito y la excelencia.

\subsection{Inteligencia emocional y el modelo de cuatro ramas}

Durante los años posteriores a su aparición el concepto de IE ha sido desarrollado e investigado por múltiples teóricos y en diversos ámbitos, generando tanta expectación como ambigüedad, dada la multitud de modelos y aproximaciones teóricas (Díaz, 2013). Esta diversidad de modelos y concepciones acerca del constructo genera ambigüedad y falta de claridad, y puede llegar a ser un problema (Mayer, Salovey y Caruso, 2008). No obstante, existe un modelo con amplia aceptación a nivel académico, el modelo de Mayer y Salovey (1997), recientemente reformulado (Mayer, Caruso y Salovey, 2016). Este modelo entiende la IE como una capacidad para percibir, valorar y expresar 
las emociones; generar sentimientos que faciliten el pensamiento; comprender el conocimiento emocional; y regular las emociones promoviendo el crecimiento emocional e intelectual.

Los teóricos defienden que la IE es un conjunto de habilidades relacionadas con el procesamiento de la información emocional, y dividen dichas habilidades en cuatro ramas: $a$ ) percibir, valorar y expresar las emociones con exactitud; $b$ ) acceder o generar sentimientos que faciliten el pensamiento; $c$ ) comprender emociones y el conocimiento emocional; $d$ ) regular las emociones promoviendo un crecimiento emocional e intelectual (Mayer y Salovey, 1997). Dentro de cada rama del modelo, los autores describieron un conjunto de habilidades o áreas de resolución de problemas, que estaban nuevamente jerarquizadas, de menor a mayor dificultad: por ejemplo, en la primera rama, "Percepción, valoración y expresión de las emociones", encontramos en primer lugar la capacidad para identificar emociones en los estados físicos, sentimientos y pensamientos de uno mismo, y en segundo lugar la capacidad para discriminar entre expresiones de sentimientos sinceras e hipócritas.

\subsection{Aportaciones de la IE a la educación}

Tres décadas después de la aparición del concepto de IE son múltiples las investigaciones realizadas acerca de sus aportaciones en diversos ámbitos. La IE "ha surgido como un constructo relevante del ajuste emocional, el bienestar personal, el éxito en la vida y las relaciones interpersonales" (Fernández y Desiree Ruiz, 2008). Por un lado, se relaciona con el bienestar y la felicidad, y por otro, capacita a las personas para afrontar de forma más adaptativa las situaciones difíciles de la vida, aumentando las posibilidades de éxito y disminuyendo las de fracaso (Fernández-Berrocal et al., 2012). Las evidencias empíricas señalan que una alta IE predice una mejor salud física y mental (Martins, Ramalho y Morin, 2010), está relacionada con unas mejores y más satisfactorias relaciones sociales (Lopes et al., 2005; Sánchez-Gómez y BresoEsteve, 2018), y tiene un papel fundamental en los éxitos profesionales (Newman, Joseph y MacCann, 2010; O’Boyle et al., 2011) y académicos (Billings et al., 2014; Perez, Cejudo y Duran, 2014). 
Prestando atención al contexto educativo, la literatura evidencia la relación de la IE con distintos factores fundamentales en la vida del estudiante, tanto dentro del contexto escolar como fuera (Brackett et al., 2006). En el ámbito educativo destacan cuatro áreas en las cuales la IE tiene una gran influencia: a) el bienestar psicológico, $b$ ) las relaciones interpersonales, $c$ ) las conductas disruptivas y $d$ ) los logros académicos.

\subsection{Bienestar psicológico}

La relación entre la IE y el ajuste psicológico ha sido uno de los aspectos más estudiados hasta la fecha. Se ha evidenciado cómo los alumnos con menores puntuaciones en IE mostraban una mayor ansiedad y un mayor número de pensamientos rumiativos (Fernández, Alcaide y Ramos, 1999). Por el contrario, altos índices en IE se relacionan con un mayor bienestar subjetivo (Brackett et al., 2006; Fernández-Berrocal y Extremera, 2016), menores niveles de ansiedad social y depresión, menores niveles de rumiación, mayor autoestima y satisfacción personal (Ciarrochi, Deane y Anderson, 2002; Salovey et al., 2002). También se ha evidenciado cómo la IE es un factor protector frente a ideas suicidas y a posibles intentos (Chan y Nock, 2009). Una larga lista de investigaciones evidencia la relación entre la IE y el bienestar psicológico, siendo este un factor importante para el éxito, lo que permite una mejor adaptación y superación de estresores (Fernández et al., 2006)

\subsection{Relaciones interpersonales}

Otros de los aspectos fundamentales en el entorno educativo son las relaciones interpersonales. Altas puntuaciones en IE utilizando el MSCEIT-YV como instrumento de medida se relacionan con una mejor competencia social y con una mayor calidad en las relaciones sociales (Brackett, Warner y Bosco, 2005; Brackett et al., 2006; Lopes et al., 2005), así como con un menor número de conflictos y relaciones negativas (Lopes, Salovey y Straus, 2003) y con unas relaciones con los padres de mayor calidad (Brackett, Rivers y Salovey, 2011). La IE ofrece recursos para desarrollar relaciones interpersonales de calidad, lo 
que se resuelve como un aspecto fundamental para el éxito académico, personal y profesional, dado que en todos los ámbitos se demanda la cooperación, la colaboración y la interdependencia positiva para alcanzar los logros.

\subsection{Conductas disruptivas}

La relación de la IE con las conductas disruptivas también ha generado un alto interés entre los investigadores. Hasta la fecha se ha observado que altos índices de IE se relacionan con un menor consumo de tabaco y alcohol (Brackett, Mayer y Warner, 2004; Trinidad y Johnson, 2002). Unido a esto, parece que los adolescentes con mayor IE son más capaces de soportar la presión social, lo que facilita la reducción del consumo de estas sustancias (Trinidad y Johnson, 2002). Otro estudio realizado con adolescentes mostró que aquellos con menor IE tenían mayor riesgo de sufrir problemas relacionados con la disciplina y la conducta en la escuela (Peters, Kranzler y Rossen, 2009). Estudiantes con bajos índices de IE mostraban grandes niveles de impulsividad y desarrollaban comportamientos antisociales (Extremera y Fernández, 2003; Petrides et al., 2004). Todas estas conductas disruptivas pueden resultar ser una merma en la consecución del éxito académico, personal y profesional, así como un lastre para las relaciones de cooperación y la interdependencia positiva, e incluso para el propio bienestar y desarrollo personal.

\subsection{Rendimiento académico}

El rendimiento académico y su relación con la IE es uno de los aspectos más estudiados y que despiertan un mayor interés en los educadores. Algunos estudios muestran asociaciones positivas entre un alto índice de IE y un mejor rendimiento académico (Barchard, 2003; Brackett y Mayer, 2003), mientras que otros no muestran esta relación (O'Connor y Little, 2003; Rode et al., 2007). Ciertamente, no parece existir una gran relación entre la IE y logro académico de forma directa, pero sí se encuentra de forma indirecta al analizar otras variables fundamentales en el rendimiento, entre ellas la salud mental (Extremera y Fernández, 2003). Aunque es necesaria una mayor investiga- 
ción acerca de cómo la IE se relaciona con el rendimiento, sí que parece claro su peso sobre otros aspectos fundamentales para el buen funcionamiento de los estudiantes en la escuela (Brackett et al., 2011). Por ejemplo, aquellos alumnos con mayor puntuación en el MSCEIT-YV mostraban una menor probabilidad de tener problemas escolares como la falta de atención o experimentar actitudes negativas hacia la escuela y hacia sus profesores (Rivers et al., 2008). Resulta lógico pensar que, a través del desarrollo de las habilidades propias de la IE, se mejora el bienestar psicológico, las relaciones interpersonales y se reducen las conductas disruptivas, lo que consecuentemente genera un mejor rendimiento académico, que permitirá posteriormente aumentar las posibilidades para alcanzar el éxito académico, personal y profesional.

\subsection{Objetivos}

El presente trabajo tiene como objetivo crear un programa pedagógico para ser aplicado por docentes de educación primaria, de modo que sean ellos quienes puedan desarrollar la IE de sus alumnos. Este programa tiene como objetivo principal el desarrollo de las habilidades emocionales interpersonales e intrapersonales, del alumnado de $5 .^{\circ}$ y $6 .^{\circ}$ curso, de forma que logren conseguir las herramientas necesarias para ser conscientes de las emociones propias y ajenas, comprendiéndolas, usándolas y regulándolas con el fin de desarrollar al máximo el potencial propio y el futuro éxito académico, profesional y personal, dirigiéndose así hacia la propia excelencia.

\section{Metodología}

\subsection{Diseño y participantes}

El presente estudio sigue una metodología cualitativa, caracterizada por su carácter exploratorio, descriptivo, inductivo, cercano a los datos y no generalizable (Lukas y Santiago, 2004). El programa de inteligencia emocional presentado a través de este documento va dirigido al alumnado de $5 .^{\circ}$ y $6 .^{\circ}$ curso de educación primaria, el cual se encuentra en un rango de edades entre $10 \mathrm{y}$ 
12 años. Todas las actividades están adaptadas a su edad y a las características de la etapa educativa en la que se encuentran.

\subsection{Procedimiento}

El programa está compuesto por 24 sesiones de una duración de 45 minutos cada una de ellas, tiempo que comprende una clase en la mayoría de centros españoles de educación primaria. Las sesiones están pensadas para ser ejecutadas un día a la semana, por lo que el programa tendrá una duración de 24 semanas. Se propone utilizar el horario de tutoría o asignaturas con las que guarda estrecha relación, como lengua, dado que a través de la puesta en práctica del programa se trabajan también contenidos propios de dicha materia. Todas las sesiones se han pensado para llevarse a cabo en un aula, por lo que pueden ser desarrolladas por cualquier maestro sin necesidad de adaptaciones.

En el cuadro 1 se presenta un esquema del programa de IE. Este está dividido en 6 bloques de contenidos o habilidades íntimamente relacionados con las cuatro ramas o factores del modelo de habilidad expuesto por Mayer y Salovey (1997).

\subsection{Instrumento de evaluación}

Con el objetivo de evaluar las posibles necesidades de forma previa al desarrollo del programa, así como su eficacia en relación con el desarrollo de las habilidades propias de la IE en el grupo de alumnos, se propone el uso de MSCEIT en su versión adaptada a la población escolar (MSCEIT-YV; Mayer, Salovey y Caruso, 2014). En esta prueba de ejecución los participantes deben resolver una serie de problemas utilizando para ello habilidades propias de la IE. MSCEIT proporciona puntuaciones en cada uno de los factores o áreas de la inteligencia emocional, correspondientes con las cuatro ramas del modelo de Mayer y Salovey: $a$ ) percepción emocional, $b$ ) facilitación emocional, $c$ ) comprensión emocional y $d$ ) regulación emocional. Puntuaciones que se divi- 
den en dos áreas: experiencial (correspondientes a las rama 1 y 2, y estratégica, correspondientes a las rama 3 y 4 . Este cuestionario debería ser realizado por la totalidad del alumnado al comienzo del programa y una vez finalizado, de forma que pueda ser observado y cuantificado cualquier cambio en las puntuaciones. Se recomienda trabajar de forma específica aquellas áreas en las que el alumnado presente puntuaciones inferiores a los valores promedio ofrecidos por el manual de uso de MSCEIT-YV.

\section{CUADRO 1}

Sesiones y contenidos del programa propuesto

\begin{tabular}{|c|c|c|c|c|}
\hline$S$ & Bloque temático y sesión & Actividades & $T$ & Materiales \\
\hline \multicolumn{5}{|c|}{ Bloque 1. MOOD-METER } \\
\hline 1 & $\begin{array}{l}\text { Contenidos, objetivos y } \\
\text { actividades }\end{array}$ & Presentación del programa IE & $45^{\prime}$ & $\begin{array}{l}\text { Pizarra digital y } \\
\text { monográfico }\end{array}$ \\
\hline 2 & $\begin{array}{l}\text { Características de la } \\
\text { emoción: afecto agradable y } \\
\text { desagradable. }\end{array}$ & $\begin{array}{l}\text { ¿Agradable o desagradable? } \\
\text { ¿Cómo de agradable? } \\
\text { ¿Cómo de desagradable? }\end{array}$ & $\begin{array}{l}15^{\prime} \\
15 \\
15\end{array}$ & \multirow[t]{2}{*}{$\begin{array}{l}\text { Lápiz y goma } \\
\text { Fichas sesiones } \\
\text { Pizarra digital* }\end{array}$} \\
\hline 3 & $\begin{array}{l}\text { Características de la } \\
\text { emoción: energía alta y baja }\end{array}$ & $\begin{array}{l}\text { ¿Energía alta o baja? } \\
\text { ¿Qué intensidad? } \\
\text { Medidor emocional }\end{array}$ & $\begin{array}{l}10^{\prime} \\
10^{\prime} \\
25\end{array}$ & \\
\hline \multicolumn{5}{|c|}{ Bloque 2. ALFABETIZACIÓN EMOCIONAL } \\
\hline 4 & Emociones básicas & $\begin{array}{l}\text { ¿Cuántas emociones } \\
\text { conocemos? } \\
\text { Origen y función } \\
\text { ¿Dónde ubicamos estas } \\
\text { emociones? }\end{array}$ & $\begin{array}{l}10^{\prime} \\
15^{\prime} \\
20\end{array}$ & \multirow{5}{*}{$\begin{array}{l}\text { Lápiz y goma } \\
\text { Cartulinas de colores } \\
\text { Tijeras } \\
\text { Pegamento } \\
\text { Cinta de doble cara } \\
\text { Fichas sesiones } \\
\text { Pizarra digital* }\end{array}$} \\
\hline 5 & $\begin{array}{l}\text { Etiquetado. } \\
\text { Mapa Emocional I }\end{array}$ & $\begin{array}{l}\text { Explicación del mapa } \\
\text { emocional } \\
\text { Creación del mapa emocional }\end{array}$ & $\begin{array}{l}5 \\
40\end{array}$ & \\
\hline 6 & $\begin{array}{l}\text { Etiquetado. } \\
\text { Mapa Emocional II }\end{array}$ & Creación del mapa emocional & $45^{\prime}$ & \\
\hline 7 & $\begin{array}{l}\text { Etiquetado. } \\
\text { Mapa Emocional III }\end{array}$ & Creación del mapa emocional & $45^{\prime}$ & \\
\hline 8 & $\begin{array}{l}\text { Etiquetado. } \\
\text { Mapa Emocional IV }\end{array}$ & Creación del mapa emocional & $45^{\prime}$ & \\
\hline
\end{tabular}




\begin{tabular}{|c|c|c|c|c|}
\hline$S$ & Bloque temático y sesión & Actividades & $T$ & Materiales \\
\hline \multicolumn{5}{|c|}{ Bloque 3. PERCEPCIÓN Y EXPRESIÓN } \\
\hline 9 & $\begin{array}{l}\text { Percepción emocional } \\
\text { interpersonal }\end{array}$ & $\begin{array}{l}\text { ¿Qué sienten? } \\
\text { Cine mudo }\end{array}$ & $\begin{array}{l}20, \\
25\end{array}$ & \multirow{4}{*}{$\begin{array}{l}\text { Lápiz y goma } \\
\text { Ficha sesiones } \\
\text { Pizarra digital* }\end{array}$} \\
\hline 10 & Percibo y expreso I & $\begin{array}{l}\text { ¿Cómo le oyes? } \\
\text { ¿Qué sienten? }\end{array}$ & $\begin{array}{l}25^{\prime} \\
20^{\prime}\end{array}$ & \\
\hline 11 & Percibo y expreso II & $\begin{array}{l}\text { El teléfono emocional } \\
\text { Busca a los tuyos }\end{array}$ & $\begin{array}{l}25^{\prime} \\
20\end{array}$ & \\
\hline 12 & Percibo y expreso III & $\begin{array}{l}\text { Emociones sociales } \\
\text { La foto }\end{array}$ & $\begin{array}{l}15^{\prime} \\
30^{\prime}\end{array}$ & \\
\hline \multicolumn{5}{|c|}{ Bloque 4. FACILITACIÓN EMOCIONAL } \\
\hline 13 & Recuerdos y emociones & Recuerdos emocionales & $45^{\prime}$ & \multirow{4}{*}{$\begin{array}{l}\text { Lápiz y goma. } \\
\text { Reproductor audio } \\
\text { Ficha sesiones } \\
\text { Escrito con historias } \\
\text { tristes y emotivas } \\
\text { Escrito con chistes }\end{array}$} \\
\hline 14 & Uso de las emociones & El armario de las emociones & $45^{\prime}$ & \\
\hline 15 & Emociones y pensamiento & Siento, luego pienso & $45^{\prime}$ & \\
\hline 16 & Perspectivas emocionales & El tren de las emociones & $45^{\prime}$ & \\
\hline \multicolumn{5}{|c|}{ Bloque 5. COMPRENSIÓN EMOCIONAL } \\
\hline 17 & Juegos emocionales & $\begin{array}{l}\text { El rosco de las emociones } \\
\text { Tabú emocional }\end{array}$ & $\begin{array}{l}25^{\prime} \\
20\end{array}$ & \multirow{4}{*}{$\begin{array}{l}\text { Lápiz y goma } \\
\text { Ficha sesiones } \\
\text { Sobres y tarjetas } \\
\text { Pizarra digital* }\end{array}$} \\
\hline 18 & Juegos emocionales II & $\begin{array}{l}\text { Password } \\
\text { ¡Vamos Rafa! }\end{array}$ & $\begin{array}{l}20^{\prime} \\
25\end{array}$ & \\
\hline 19 & $\begin{array}{l}\text { Situación-Pensamiento- } \\
\text { Emoción }\end{array}$ & Siento, luego pienso & $45^{\prime}$ & \\
\hline 20 & El lugar del otro & Juego de roles & $45^{\prime}$ & \\
\hline \multicolumn{5}{|c|}{ Bloque 6. REGULACIÓN EMOCIONAL } \\
\hline 21 & Regulando emociones & ¿Cómo regulo mis emociones? & $45^{\prime}$ & \multirow{4}{*}{$\begin{array}{l}\text { Lápiz y goma } \\
\text { Fichas sesiones } \\
\text { Pizarra digital* }\end{array}$} \\
\hline 22 & Influyendo en los otros & $\begin{array}{l}\text { ¿Qué me hacen sentir? } \\
\text { Relaja tu cuerpo }\end{array}$ & $\begin{array}{l}25^{\prime} \\
20\end{array}$ & \\
\hline 23 & $\begin{array}{l}\text { Manejando emociones en } \\
\text { otros }\end{array}$ & $\begin{array}{l}\text { Manejo sus emociones } \\
\text { Regulando que es gerundio }\end{array}$ & $\begin{array}{l}25, \\
20\end{array}$ & \\
\hline 24 & Inteligente emocional & $\begin{array}{l}\text { Soy inteligente emocional } \\
\text { Cierre de programa }\end{array}$ & $\begin{array}{l}35^{\prime} \\
10^{\prime}\end{array}$ & \\
\hline
\end{tabular}

$\mathrm{S}=$ número de sesión, $\mathrm{T}=$ tiempo en minutos. 


\section{REsultados}

\subsection{Contenido del bloque I. Mood-Meter}

El bloque I de contenidos está formado por tres sesiones, las cuales tienen como objetivo presentar el programa e introducir el mood-meter (medidor emocional), una herramienta fundamental para la identificación de las emociones propias que se utilizará durante toda la formación. Durante las sesiones 2 y 3, los alumnos conocerán el modelo circumplejo de Russell (1980), el cual propone que la emoción está compuesta por dos dimensiones: energía, entendida como alta/baja, y afecto, entendido como agradable/desagradable. De este modelo nace el medidor emocional, un sistema de coordenadas dividido en cuatro cuadrantes en el cual se ubica todo el espectro de emociones que podemos llegar a sentir (figura 1).

\section{FIGURA 1}

Medidor emocional basado en el modelo de Russell (1980)

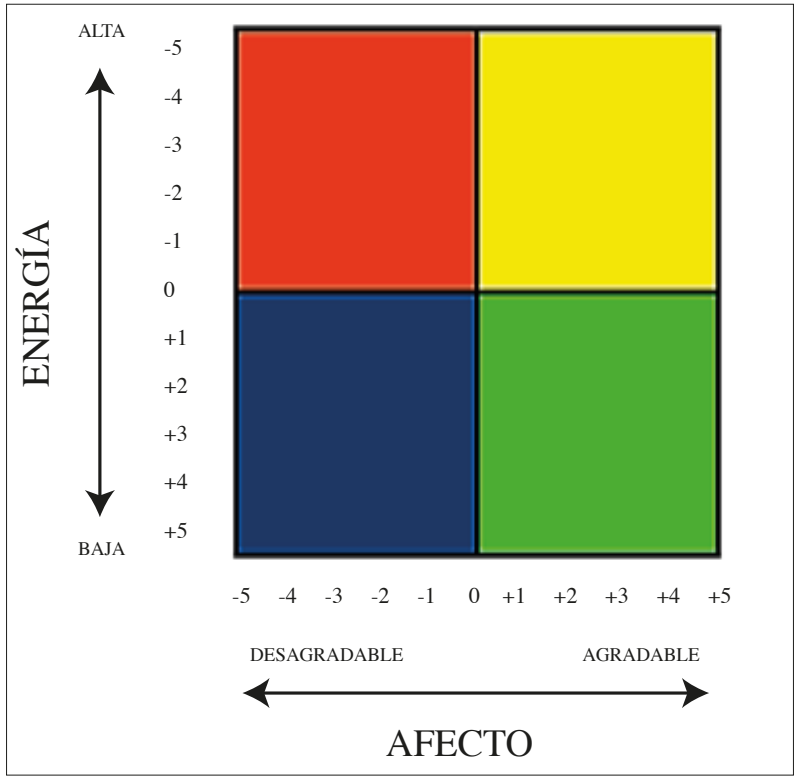

Fuente: elaboración propia. 
La habilidad para identificar y percibir emociones supone el primer bloque de la IE, por eso se sitúan estas sesiones al comienzo del programa. Además, dado que es un programa de formación pensado y diseñado para llevarse a cabo en el aula, el aprendizaje y uso del medidor emocional se debe extraer e incluir en la dinámica de aula, de forma que durante el transcurso de las sesiones escolares el grupo de alumnos utilice esta herramienta, especialmente en momentos clave como el comienzo y el fin del día escolar, así como la salida al recreo y la posterior vuelta.

\subsection{Contenido del bloque II. Alfabetización emocional}

El segundo bloque de contenidos, Alfabetización emocional, está constituido por cinco sesiones. En la primera, los alumnos conocerán cuáles son las emociones básicas (alegría, sorpresa, miedo, asco, tristeza, ira), por qué aparecen y qué función tienen (tabla 1).

TABLA 1

Emociones básicas

\begin{tabular}{|l|l|l|}
\hline \multicolumn{1}{|c|}{ Emoción } & \multicolumn{1}{|c|}{ Motivo de aparición } & \multicolumn{1}{c|}{ Función } \\
\hline Alegría & Consecución de algo deseado. & $\begin{array}{l}\text { Cohesión, seguridad, sensación } \\
\text { de bienestar. }\end{array}$ \\
\hline Sorpresa & Estímulo inesperado. & $\begin{array}{l}\text { Facilita la atención y exploración } \\
\text { del entorno. }\end{array}$ \\
\hline Miedo & Situaciones o estímulos de carácter amenazante. & $\begin{array}{l}\text { Protegernos física y } \\
\text { psicológicamente. }\end{array}$ \\
\hline Asco & $\begin{array}{l}\text { Estímulos que nos resultan aversivos o } \\
\text { desagradables. }\end{array}$ & $\begin{array}{l}\text { Rechazo y distancia de aquello } \\
\text { que puede resultar nocivo. }\end{array}$ \\
\hline Tristeza & $\begin{array}{l}\text { Percepción de una pérdida o vivencia de un } \\
\text { suceso desagradable. }\end{array}$ & $\begin{array}{l}\text { Recuperación personal, motiva } \\
\text { a pedir ayuda y aumentar los } \\
\text { recursos. }\end{array}$ \\
\hline Ira & $\begin{array}{l}\text { No consecución de objetivos o interferencia de } \\
\text { personas/situaciones en ellos. }\end{array}$ & $\begin{array}{l}\text { Autodefensa y motivación para } \\
\text { la acción. }\end{array}$ \\
\hline
\end{tabular}

En las cuatro sesiones restantes, tomando como referencia el medidor emocional ya estudiado, los alumnos crearán un "mapa emocional”, en el cual se 
incluirán alrededor de 100 etiquetas emocionales, las cuales representarán el vocabulario emocional de la clase (figura 2). Para empezar, se recomienda situar las emociones de los extremos, de forma que sirvan como referencia. Por ejemplo, en el extremo superior izquierdo (energía alta y afecto negativo) se puede situar la ira, en el inferior izquierdo la decepción (energía baja y afecto negativo), en el extremo superior derecho la euforia (energía alta y afecto positivo), mientras que en el margen inferior derecho puede colocarse la satisfacción (energía baja y afecto positivo). La habilidad para etiquetar de forma precisa las emociones y conocer las relaciones entre estas es la primera habilidad dentro de la rama de comprensión emocional, y se trata de una habilidad imprescindible, ya que la adquisición de un vocabulario emocional rico y diverso permitirá una mejor comprensión y expresión tanto de las emociones propias como ajenas. Tal es la importancia de tener un amplio vocabulario, especialmente en educación primaria, que esta habilidad ha sido extraída del bloque que le correspondería según el modelo de cuatro ramas, "comprensión emocional", para tener su propio bloque y trabajarse en profundidad, asegurando la creación de un vocabulario emocional preciso y diverso. La adquisición temprana de un amplio vocabulario emocional en el grupo permitirá una mejor adquisición de los aprendizajes propios de los bloques posteriores.

FIGURA 2

Ejemplo de mapa emocional para completar

\begin{tabular}{|l|l|l|l|l|l|l|l|l|l|}
\hline & & & & & & & & & \\
\hline & & & & & & & & & \\
\hline & & & & & & & & & \\
\hline & & & & & & & & & \\
\hline & & & & & & & & & \\
\hline & & & & & & & & & \\
\hline & & & & & & & & & \\
\hline & & & & & & & & & \\
\hline & & & & & & & & & \\
\hline & & & & & & & & & \\
\hline
\end{tabular}




\subsection{Contenido del bloque III. Percepción emocional}

El bloque III, percepción emocional, está constituido por cuatro sesiones, en las cuales se trabajarán las habilidades de percepción de las emociones propias, identificación de las emociones en otros, percepción de las emociones en el ambiente y expresión de las emociones de forma adecuada, todas ellas habilidades de la IE ubicadas en la primera rama del modelo de Mayer y Salovey.

Una de las actividades propuestas en el bloque es la denominada "Cine mudo", en la que se trabajarán las habilidades de percepción a través de juegos basados en expresión corporal. En dicha actividad, el grupo aula se dividirá en grupos de 4-5 integrantes. A cada grupo se le asignará una emoción social ya trabajada con anterioridad (por ejemplo, vergüenza). Los integrantes tendrán un tiempo limitado (5'-10') para preparar una escena muda, en la que solo pueden expresar a través del cuerpo, sin hablar, y en la que se transmita de forma conjunta la emoción dada para que el resto de grupos la intenten adivinar.

El objetivo principal de este bloque es que los alumnos sean capaces de identificar las emociones que sienten los otros a través de la expresión facial, el tono de voz y la conducta; así como identificar las emociones que se despliegan a distintos ambientes; y expresar las emociones de forma efectiva y adecuada.

\subsection{Contenido del bloque IV: Facilitación emocional}

En el cuarto bloque de contenidos, constituido nuevamente por cuatro sesiones, se trabajarán las habilidades de la IE correspondientes a la segunda rama del modelo, facilitación emocional. La facilitación del pensamiento usando las emociones implica generar emociones que faciliten el juicio y la memoria o aprovechar el cambio de humor para generar distintas perspectivas cognitivas. Dicho bloque se centra en la idea de que las emociones facilitan e influyen en el pensamiento y en el modo de pensar. El objetivo principal es, por tanto, que los alumnos sean conscientes de la relación entre las emociones y el pensamiento, y empleen este conocimiento para conseguir sus objetivos. 
Una de las actividades propuestas es "El tren de las emociones". En esta actividad se invita al alumnado a realizar un viaje en tren por distintos mundos emocionales, que simbolizan los distintos estados emocionales repartidos por el medidor emocional. En cada mundo, el docente guiará a los alumnos para que, utilizando su imaginación, sientan y conecten con la emoción dada. Los alumnos tendrán que pensar un problema que tengan en su vida diaria, a poder ser con algún compañero, y que aún no hayan resuelto. Durante el paso por los distintos mundos emocionales, los alumnos de forma individual tendrán que contestar a una serie de preguntas desde la perspectiva de dicha emoción. Algunas de las preguntas son:

Desde esta emoción... ¿Qué pensarías? ¿Qué harías? ¿Qué sentiría la otra persona? ¿Cómo reaccionaría la otra persona? ¿Cómo te sentirías después de hacerlo?

El objetivo de la actividad es que los alumnos entiendan que los distintos estados emocionales facilitan distintos tipos de pensamiento y razonamiento ante el mismo problema, y que pueden utilizar las distintas emociones para descubrir diferentes perspectivas ante una misma situación, y posteriormente tomar la decisión que consideren más conveniente.

\subsection{Contenido del bloque V: Comprensión emocional}

A lo largo del quinto bloque se desarrollan las competencias integradas en la tercera rama del modelo de Mayer y Salovey, la comprensión emocional. Dentro de este bloque los alumnos aprenderán a inferir los antecedentes, el significado y las consecuencias de las emociones; evaluarán las situaciones que pueden provocar las emociones; tratarán de comprender las emociones mixtas y complejas; reconocerán las probables transiciones entre emociones y la previsión emocional, es decir, comprender cómo se puede sentir una persona en un futuro o bajo ciertas circunstancias.

Una de las actividades principales es "Siento, luego pienso". Esta se divide en tres tareas, secuenciadas de menor a mayor dificultad. El objetivo es que los alumnos entiendan la relación entre situación -pensamiento- y emoción, y cómo nuestra forma de pensar puede generar distintas emociones. Así pues, se crearán grupos de 4-5 alumnos y a cada uno se les repartirá una serie de 
sobres con tarjetas. En la tarea 1, la tarjeta incluirá una situación, tres formas de pensar y tres emociones, y los alumnos tendrán que decir que emoción corresponde a cada forma de pensar. En la tarea 2, se les presentará a los alumnos una situación y tres emociones, y estos tendrán que inventar una forma de pensar que genere cada una de las emociones. Por último, en la tarea 3, se dará a los alumnos una situación y tres emociones que aparecen mezcladas en esa situación, y los alumnos tendrán que explicar por qué aparecen estas emociones.

\subsection{Contenido del bloque VI: Regulación emocional}

El bloque VI de contenidos, constituido por cuatro sesiones, será el último del programa y permitirá al alumnado desarrollar habilidades integradas dentro de la cuarta rama del modelo, regulación emocional. En este bloque se desarrollarán habilidades tales como: la capacidad para aceptar sentimientos tanto placenteros como desagradables; la capacidad para reconocer la utilidad de las emociones; el análisis y determinación de estrategias de regulación y la habilidad para regular emociones propias y ajenas. Esta habilidad de regulación, a su vez, es una habilidad que incluye y depende de todas las anteriores, ya que como exponen los autores del modelo es necesaria la percepción, uso, comprensión y expresión emocional para llegar a la regulación reflexiva. El objetivo del bloque es que los alumnos integren todas las anteriores habilidades y sean conscientes de la importancia de la regulación emocional para el desarrollo personal y social. Para concluir el bloque y el programa, se propone una actividad que aúna todos los contenidos trabajados y se titula "Soy inteligente emocional". La actividad consiste en proyectar una escena de una película, escogida por el docente, en la que dos personajes discutan. Los alumnos tendrán que fijarse en cada personaje, en su expresión y reacción emocional, y utilizar los conocimientos adquiridos durante el programa para razonar acerca de la interacción que tiene lugar en la escena y así rellenar una ficha contestando una serie de preguntas (véase figura 3). El objetivo es que, ante una situación dada, como una escena de una película, los alumnos sepan aplicar la percepción, facilitación, comprensión y regulación emocional, habilidades adquiridas durante el programa. 
FIGURA 3

Ficha para completar la actividad "Soy inteligente emocional"

\begin{tabular}{|l|l|}
\hline \multicolumn{2}{|c|}{ Personaje 1 } \\
\hline ¿Qué emoción expresa? & \\
\hline ¿Qué crees que piensa? & \\
\hline ¿Qué hace? & \\
\hline ¿Qué crees que siente realmente? & \\
\hline $\begin{array}{l}\text { ¿Es adecuada la reacción que tiene ante la } \\
\text { situación? }\end{array}$ & \\
\hline $\begin{array}{l}\text { ¿Cómo habrías reaccionado tú y cómo piensas } \\
\text { que se debería reaccionar? }\end{array}$ & \\
\hline & Personaje 2 \\
\hline ¿Qué emoción expresa? & \\
\hline ¿Qué crees que piensa? & \\
\hline ¿Qué hace? & \\
\hline ¿Qué crees que siente realmente? & \\
\hline $\begin{array}{l}\text { ¿Es adecuada la reacción que tiene ante la } \\
\text { situación? }\end{array}$ \\
\hline $\begin{array}{l}\text { ¿Cómo habrías reaccionado tú y cómo piensas } \\
\text { que se debería reaccionar? }\end{array}$ & \\
\hline
\end{tabular}

\section{Discusión y CONCLUSIONES}

Integrar las habilidades socioemocionales en el aula se ha convertido en una necesidad, tanto educativa como social. En los últimos años, el desarrollo de las habilidades propias de la IE se ha vuelto un elemento fundamental para explicar el desarrollo personal y socioemocional de los estudiantes (Fernández-Berrocal y Aranda, 2017). Un gran número de investigaciones, como se ha expuesto a lo largo del presente trabajo, muestran los positivos beneficios del desarrollo de la IE en la escuela, y recientemente se ha evidenciado que la IE se puede mejorar a través de intervenciones educativas aplicadas por el docente (Hodzic et al., 2018). Sin embargo, pese a que más de la mitad del 
cuerpo docente no ha sido formado en habilidades emocionales (Fernández, Sánchez y García, 2019), es importante remarcar el papel fundamental del profesorado a la hora de integrar estas habilidades en el aula (ExtremeraPacheco, Mérida-López y Sánchez-Gómez, 2019).

El principal resultado de esta investigación es un programa pedagógico práctico diseñado de forma útil y sencilla para poder ser llevado a cabo en cualquier aula de $5 .^{\circ}$ y $6 .^{\circ}$ de Educación Primaria. A lo largo del trabajo se pretende ofrecer a todo docente sin formación previa un apoyo esencial a la hora de llevar a cabo un programa de desarrollo de la IE en la escuela, aportando el conocimiento esencial acerca de la IE, el modelo de Mayer y Salovey y los instrumentos de medida necesarios para evaluar la necesidad de aplicación y el impacto de la formación.

A través del actual trabajo se despliega un programa de desarrollo de la inteligencia emocional formado por 24 sesiones, que incluye distintos bloques de contenidos, relacionados con las habilidades que integran la IE según el modelo de habilidad. Se trata de un programa que aún no ha sido desarrollado, por lo que no desprende resultados acerca de su eficacia. Independientemente de este hecho, se trata de una propuesta basada en la contrastada literatura previa, la cual aporta un importante respaldo científico. Como se ha descrito en la introducción, la IE debe ser considerada como un conjunto de habilidades mejorables, por tanto, a la hora de generar y aplicar programas para la mejora y el desarrollo de la IE, tomar el modelo de habilidad de cuatro ramas de Mayer y Salovey se resuelve como el método más idóneo en esta empresa. Además, este modelo proporciona una aproximación conceptual coherente y defiende que el constructo coincide con los criterios tradicionales de inteligencia (Mayer, Robers y Barsade, 2008; Mayer, Salovey y Caruso, 2008), y permite evaluar la IE utilizando distintos procedimientos, con lo que aporta un enfoque sólido y estable, que facilita la creación de programas para su desarrollo e instrumentos que puedan medirlo (Extremera y FernándezBerrocal, 2003).

En conclusión, el presente trabajo aporta un nuevo modo de desarrollar la IE en el alumnado de último ciclo de Educación Primaria, con la novedad de haber sido conceptualizado y desarrollado para que sea el propio docente, sin necesidad de tener formación previa, el encargado de llevar a cabo la formación. El correcto desarrollo del programa espera otorgar a los alumnos 
herramientas para desarrollar y mejorar sus habilidades emocionales inter e intrapersonales, favoreciendo y accionando así el descubrimiento y desarrollo de sus talentos y potencialidades, dirigiéndolos hacia el éxito y la excelencia.

Conflicto de intereses

Los autores de este trabajo declaran que no existe conflicto de intereses.

\section{Financiación}

Este trabajo ha sido cofinanciado por la Generalitat Valenciana y el Fondo Social Europeo (ACIF / 2017/201).

\section{BiBLIOGRAFÍA}

BARChARD, K. A. (2003). Does emotional intelligence assist in the prediction of academic success? Educational and psychological measurement 63(5), 840-858. DOI: https://doi.org/10.1177/0013164403251333.

Belfield, C., Bowden, A. B., Klapp, A., Levin, H., Shand, R. y Zander, S. (2015). The economic value of social and emotional learning. Journal of Benefit-Cost Analysis 6(3), 508-544.

Billings, C. E., Downey, L. A., Lomas, J. E., Lloyd, J. y Stough, C. (2014). Emotional Intelligence and scholastic achievement in pre-adolescent children. Personality and Individual Differences 65, 14-18. DOI: https://doi. org/10.1016/j.paid.2014.01.017.

Brackett, M. A. y Mayer, J. D. (2003). Convergent, discriminant, and incremental validity of competing measures of emotional intelligence. Personality and social psychology bulletin 29(9), 1147-1158. DOI: https://doi. org/10.1177/0146167203254596.

Brackett, M. A., Mayer, J. D. y Warner, R. M. (2004). Emotional intelligence and its relation to everyday behaviour. Personality and Individual differences 36(6), 1387-1402. DOI: https://doi.org/10.1016/S01918869(03)00236-8. 
Brackett, M. A., Rivers, S. E. y Salovey, P. (2011). Emotional intelligence: Implications for personal, social, academic, and workplace success. Social and Personality Psychology Compass 5(1), 88-103. DOI: https://doi. org/10.1111/j.1751-9004.2010.00334.x.

Brackett, M. A., Rivers, S. E., Shiffman, S., Lerner, N. y Salovey, P. (2006). Relating emotional abilities to social functioning: a comparison of self-report and performance measures of emotional intelligence. Journal of personality and social psychology 91(4), 780-795. DOI: https://doi. org/10.1037/0022-3514.91.4.780.

Brackett, M. A., Warner, R. M. y Bosco, J. S. (2005). Emotional intelligence and relationship quality among couples. Personal relationships 12(2), 197-212. DOI: 10.1111/j.1350-4126.2005.00111.x

CAmusso, P. A. (2019). ¿Cuáles son algunas de las características de la modernidad líquida que ponen en tensión cómo seducir a los estudiantes? Voces de la Educación 4(8), 17-27.

Ciarrochi, J., Deane, F. P. y Anderson, S. (2002). Emotional intelligence moderates the relationship between stress and mental health. Personality and individual differences 32(2), 197-209. DOI: https://doi.org/10.1016/ S0191-8869(01)00012-5.

Chan, C. B. y Nock, M. K. (2009). Emotional Intelligence is a Protective Factor for Suicidal Behavior. Journal of the American Academy of Child and Adolescent Psychiatry 48, 422-430. DOI: https://doi.org/10.1097/ CHI.0b013e3181984f44.

DíAz, J. J. M. (2013). Reseña teórica de la inteligencia emocional: modelos e instrumentos de medición. Revista científica 1(17), 10-32. DOI: https:// doi.org/10.14483/23448350.4505.

Elias, M. J., Arnold, H. y Steiger-Hussey, C. (2003). EQ+IQ=Best Leadership Practices for Caring and Successful Schools. California: Corwin Press Inc.

Extremera, N. y Fernández-Berrocal, P. (2003). La inteligencia emocional en el contexto educativo: hallazgos científicos de sus efectos en el aula. Revista de educación 332(12), 97-116.

Extremera Pacheco, N., Mérida López, S. y Sánchez Gómez, M. (2019). La importancia de la inteligencia emocional del profesorado en la misión 
educativa: impacto en el aula y recomendaciones de buenas prácticas para su entrenamiento. Voces De La Educación, 74-97.

Fernández, A. B., SÁnchez, R. y García, A. A. (2019). Formación emocional del profesorado y gestión del clima de su aula. Praxis \& Saber 10(24), 119-141.

Fernández-Berrocal, P., Alcaide, R., Extremera, N. y Pizarro, D. (2006). The role of emotional intelligence in anxiety and depression among adolescents. Individual Differences Research 4(1), 16-27.

Fernández-Berrocal, P., Alcaide, R. y Ramos, N. (1999). The influence of emotional intelligence on the emotional adjustment in highschool students. Bulletin of Kharkov State University 439(1-2), 119-123.

Fernández-Berrocal, P. y Aranda, D. R. (2017). La inteligencia emocional en la educación. Electronic Journal of Research in Education Psychology 6(15), 421-436. DOI: http://dx.doi.org/10.25115/ejrep.v6i15.1289.

Fernández-Berrocal, P., Berrios-Martos, M. P., Extremera, N. y Augusto, J. M. (2012). Inteligencia emocional: 22 años de avances empíricos. Psicología Conductual 20(1), 5-13.

Fernández-Berrocal, P. y Extremera, N. (2016). Ability Emotional Intelligence, Depression, and Well-Being. Emotion Review 8(4), 311-315. DOI: https://doi.org/10.1177/1754073916650494.

Fernández-Berrocal, P. y Ruiz, D. (2008). Emotional intelligence in education. Electronic Journal of Research in Educational Psychology 15(6), 421-436.

GARDNER, H. (1983). Frames of mind: The theory of multiple intelligences. New York: Basic Books.

Hodzic, S., Scharfen, J., Ripoll, P., Holling, H. y Zenasni, F. (2018). How Efficient are Emotional Intelligence Trainings: A meta-Analysis. Emotion Review 10(2), 138-148. DOI: https://doi.org/10.1177/1754073917708613. Lopes, P. N., Salovey, P., Côté, S., Beers, M. y Petty, R. E. (2005). Emotion Regulation Abilities and the Quality of Social Interaction. Emotion 5(1), 113-118. DOI: https://doi.org/10.1037/1528-3542.5.1.113.

Lopes, P. N., Salovey, P. y Straus, R. (2003). Emotional intelligence, personality, and the perceived quality of social relationships. Personality and individual Differences 35(3), 641-658. DOI: https://doi.org/10.1016/ S0191-8869(02)00242-8. 
Lukas, J. F., y Santiago, K. (2004). Evaluación educativa. Madrid: Alianza Editorial.

Martins, A., Ramalho, N. y Morin, E. (2010). A comprehensive meta-analysis of the relationship between emotional intelligence and health. Personality and Individual Differences 49(6), 554-564. DOI: https://doi. org/10.1016/j.paid.2010.05.029.

Mayer, J. D., Caruso, D. R. y Salovey, P. (2016). The Ability Model of Emotional Intelligence: Principles and Updates. Emotion review 8(4), 290300. DOI: https://doi.org/10.1177/1754073916639667.

Mayer, J. D., Roberts, R. D. y Barsade, S. G. (2008). Emerging research in Emotional Intelligence. Annual Review of Psychology 59, 507-536. DOI: https://doi.org/10.1146/annurev.psych.59.103006.093646.

Mayer, J. D. y Salovey, P. (1997). What is emotional intelligence?, en P. Salovey y D. Sluyter (eds.), Emotional Development and Emotional Intelligence: Implications for Educators (pp. 3-31). New York: Basic Books.

Mayer, J. D., Salovey, P. y Caruso, D. R. (2008). Emotional intelligence: New ability or eclectic traits? American Psychologist 63(6), 503-517. DOI: https://doi.org/10.1037/0003-066X.63.6.503.

Mayer, J. D., Salovey, P. y Caruso, D. R. (2014). Mayer-Salovey-Caruso Emotional Intelligence Test: Youth Research Version, MSCEIT: YRV. Toronto, Ontario: MHS.

Newman, D. A., Joseph, D. L. y MacCann, C. (2010). Emotional intelligence and Job Performance: The Importance of Emotion Regulation and Emotional Labor Context. Industrial and Organizational Psychology 3(2), 159-164. DOI: https://doi.org/10.1111/j.1754-9434.2010.01218.x.

O’Boyle Jr, E. H., Humphrey, R. H., Pollack, J. M., Hawver, T. H. y StoRY, P. A. (2011). The relation between emotional intelligence and job performance: A meta-analysis. Journal of Organizational Behavior 32(5), 788-818. DOI: https://doi.org/10.1002/job.714.

O’Connor Jr, R. M. y LittLe, I. S. (2003). Revisiting the predictive validity of emotional intelligence: Self-report versus ability-based measures. Personality and Individual differences 35(8), 1893-1902. DOI: https://doi. org/10.1016/S0191-8869(03)00038-2.

Perez-Gonzalez, J. C., Cejudo-Prado, M. J. y Duran-Arias, C. R. (2014). Emotional intelligence as a non-cognitive predictor of academic perfor- 
mance. Personality and Individual Differences 60, S30. DOI: https://doi. org/10.1016/j.paid.2013.07.053.

Peters, C., Kranzler, J. H. y Rossen, E. (2009). Validity of the Mayer -Salovey- Caruso emotional intelligence test: youth version -research edition. Canadian Journal of School Psychology 24(1), 76-81. DOI: https:// doi.org/10.1177/0829573508329822.

Petrides, K. V., Frederickson, N. y Furnham, A. (2004). The role of trait emotional intelligence in academic performance and deviant behavior at school. Personality and individual differences 36(2), 277-293. DOI: https://doi.org/10.1016/S0191-8869(03)00084-9.

Rivers, S. E., Brackett, M. A. y Salovey, P. (2008). Measuring emotional intelligence as a mental ability in adults and children. The Sage handbook of personality theory and assessment 2, 440-460.

Rode, J. C., Mooney, C. H., Arthaud-Day, M. L., Near, J. P., Baldwin, T. T., Rubin, R. S. y Bommer, W. H. (2007). Emotional intelligence and individual performance: Evidence of direct and moderated effects. Journal of Organizational Behavior 28(4), 399-421. DOI: https://doi.org/10.1002/ job.429.

Russell, J. A. (1980). A circumplex model of affect. Journal of personality and social psychology 39(6), 1161-1178. DOI: https://doi.org/10.1037/ h0077714.

Salovey, P., Stroud, L. R., Woolery, A. y Epel, E. S. (2002). Perceived Emotional Intelligence, Stress Reactivity, and Symptom Reports: Further Explorations Using the Trait Meta-Mood Scale. Psychology and health 17(5), 611-627. DOI: https://doi.org/10.1080/08870440290025812.

Salovey, P. y Mayer, J. D. (1990). Emotional Intelligence. Imagination, Cognition and Personality 9(3), 185-211. DOI: https://doi.org/10.2190/ DUGG-P24E-52WK-6CDG.

SAnchez-Gomez, M. y Breso-Esteve, E. (2018). Inteligencia emocional para frenar el rechazo en las aulas. Agora de salut 5, 275-282. DOI: http:// dx.doi.org/10.6035/AgoraSalut.2018.5.30.

Trinidad, D. R. y Johnson, C. A. (2002). The association between emotional intelligence and early adolescent tobacco and alcohol use. Personality and individual differences 32(1), 95-105. DOI: https://doi.org/10.1016/S01918869(01)00008-3. 
VALLÉs, A. (2001). La inteligencia emocional y sus habilidades. Bases Neuroanatómicas. III Jornadas de Innovación Pedagógica: Inteligencia Emocional, 22-45.

Weissberg, R. P., Durlak, J. A., Domitrovich, C. E. y Gullotta, T. P. (eds.) (2015). Social and emotional learning: Past, present, and future, en J. A. Durlak, C. E. Domitrovich, R. P. Weissberg, y T. P. Gullotta (eds.), Handbook of social and emotional learning: Research and practice (pp. 3-19). New York: The Guilford Press. 